\title{
Lecturas de la Obra de John Dewey en Intersección con el Catolicismo: Los casos de la Institución Libre de Enseñanza y la Tesis sobre Dewey del Padre Alberto Hurtado, S. J.
}

\section{The Readings of John Dewey's Work and the Intersection of Catholicism: The Cases of the Institución Libre de Enseñanza and the Thesis of Father Alberto Hurtado, S.J. on Dewey}

\author{
Les lectures de l'œuvre de John Dewey et \\ I'intersection du Catholicisme: Les cas de \\ I'Institution libre de l'enseignement et la thèse \\ du Père Alberto Hurtado, S.J. sur Dewey
}

\author{
Rosa Bruno-Jofré \\ Queen's University, Canadá
}

\section{Gonzalo Jover}

Universidad Complutense de Madrid, España

\section{RESUMEN}

Este artículo examina el modo en que la intersección con el catolicismo afectó las lecturas que se hicieron de Dewey y de la educación progresiva en dos diferentes escenarios hispano parlantes. El primer escenario se refiere a cómo fue leído Dewey, en España, por la Institución Libre de Enseñanza, en el contexto del debate religioso que tuvo lugar en este país en las décadas en torno al cambio de siglo. El segundo, trata de la lectura que hizo de Dewey el Padre Alberto Hurtado (recientemente canonizado), un sacerdote jesuita chileno, graduado en Lovaina en 1935, y fundador del Hogar de Cristo, quien gozó de una importante presencia educativa en Chile. La intersección con el catolicismo fue relevante en los dos escenarios, aunque las 
mediaciones fueron sustancialmente diferentes. Ambas lecturas separaron deliberadamente el pensamiento pedagógico de Dewey de su filosofía pragmatista. Sin embargo, mientras el Padre Hurtado hizo una lectura católica para articular el pensamiento pedagógico de Dewey con la trascendencia religiosa, los miembros de la Institución leyeron a Dewey con la intención de liberar a la educación del dogmatismo religioso, pero manteniendo una visión trascendental del ser humano. Los dos escenarios muestran, no sólo que los sistemas de creencias y hábitos ponen límites a lo que puede ser integrado en una concepción de la educación, sino, también, la importancia de las condiciones contextuales en la articulación de las teorías y las prácticas educativas.

Descriptores: Dewey y Alberto Hurtado; Dewey y la Institución de Libre Enseñanza; Dewey y religión; transcendencia y Dewey; Dewey en Chile; Dewey en España; democracia, experimentalismo, y educación.

\section{ABSTRACT}

This paper examines the way the intersections with religion affected the readings of Dewey and progressive education themes in two discursive Spanish-speaking scenarios. The first scenario refers to how Dewey was read by the Spanish Institución Libre de Enseñanza within the context of the religious (Catholic) debate that took place in Spain during the first decade of the twentieth century. The second refers to the reading of Dewey by Father Alberto Hurtado (recently canonized), a Chilean Jesuit priest, a graduate from Louvain in 1935, and founder of the Hogar de Cristo, who had an important educational presence in Chile. The intersection of Catholicism was relevant in the two scenarios although the mediations were substantially different. Both readings purposely separated Dewey's pedagogical thinking from his pragmatist philosophy. However, while Father Hurtado did a Catholic reading to articulate Dewey's pedagogical thinking with religious transcendence, the members of the Institución read Dewey with the intention of liberating education from religious dogmatism, but still keeping a transcendental vision of the humane. The two scenarios show not only that habits and belief systems set limits on what can be integrated in a concept of education, but also the relevance of contingent conditions in the articulation of educational theories and practices.

Key words: Dewey and Alberto Hurtado; Dewey and the Free Teaching Institute; Dewey and religion; transcendence and Dewey; Dewey in Chile; Dewey in Spain; democracy, experimentalism, and education.

\section{RÉSUMÉ}

Ce papier examine dans deux scénarios discursifs de langue espagnole comment les intersections avec la religion influèrent sur les lectures de Dewey et les thèmes de l'éducation progressiste. Le premier scénario se réfere à la façon dont Dewey a été lu par l'Institution libre de l'enseignement dans le contexte du débat religieux (catholique) qui eut lieu en Espagne au cours de la première décennie du vingtième siècle. Le second vise la lecture de Dewey par le Père Alberto Hurtado (canonisé récemment) un prêtre jésuite chilien, diplômé de Louvain en 1935, et fondateur de Hogar de Cristo qui fut une présence éducative importante au Chili. L'intersection du Catholicisme fut significative dans les deux scénarios quoique les médiations furent substantiellement différentes. Les deux lectures séparèrent intentionnellement la pensée pédagogique de Dewey de sa philosophie pragmatiste. Toutefois, tandis que le Père Hurtado en a fait une lecture " catholique " pour articuler la pensée pédagogique de Dewey avec une transcendance religieuse, les membres de l'Institution ont lu Dewey avec l'intention de libérer l'éducation du dogmatisme religieux, tout en gardant une vision transcendantale de l'humain. Les deux scénarios montrent que, non seulement les habitudes et les systèmes de croyance posent des limites à ce qui peut être intégré dans un concept de l'éducation, mais montre aussi la pertinence des conditions contingentes dans l'articulation des théories et des pratiques éducatives.

Mots clés : Dewey et Alberto Hurtado, Dewey et I'Institut de l'enseignement libre; Dewey et la religion; La transcendance et Dewey; Dewey au Chili; Dewey en Espagne; La démocratie, I'expérimentalisme et l'éducation 


\section{Introducción *}

E n su estudio sobre la recepción de las ideas de John Dewey en los países de Chabla hispana, señala Donoso que "Dewey atrajo, en Latinoamérica, lo mismo que en España, a aquellos educadores que eran anti-tradicionalistas en sus prácticas educativas, generalmente liberales en cuanto a las reformas político-sociales y defensores de las tradiciones seculares (lo que, en muchos casos, significaba ser anticlericales)." ${ }^{1}$ Este fue en gran medida el caso de la Institución Libre de Enseñanza, una organización educativa fundada por Francisco Giner de lo Ríos en 1876, con el objetivo de liberar la educación del Estado y de la Iglesia Católica, que abrazó el liberalismo político, la filosofía de Krause y las ideas pedagógicas de Froebel. A principios del siglo veinte, la Institución vio en Dewey una fuente de inspiración para la innovación educativa y la transformación social. Sin embargo, la difusión y articulación de las ideas de Dewey en los diferentes espacios históricos no siguieron una trayectoria única. La lectura que el jesuita chileno Alberto Hurtado (1901-1952) hizo de John Dewey en su Tesis doctoral, defendida en 1935 en la Universidad Católica de Lovaina (Bélgica), nos ofrece un ejemplo sólido. El Padre Hurtado leyó a Dewey a través de las lentes de la doctrina de la Iglesia Católica y del neo-tomismo, teñidas de su propia visión de la espiritualidad católica, su búsqueda de una visión de lo humano y su apasionado compromiso con el cambio social. La presencia en el escenario político chileno del momento de las nuevas ideas acerca de la educación y de las obras de John Dewey, en medio de la agitación política y social, movieron a Hurtado a realizar una lectura alternativa de Dewey, cuyas ideas filosóficas no eran admisibles para la jerarquía eclesiástica. ${ }^{2}$ En este sentido, la École de pedagogie et de psychologie appliquée à l'éducation de la Universidad Católica de Lovaina, fundada en 1923, le proporcionó el contexto intelectual católico. ${ }^{3} \mathrm{La}$ interpretación que hizo Hurtado de las ideas de Dewey llegó a una audiencia amplia, a través de los artículos que escribió, los seminarios públicos, las clases y su implicación con el Colegio de San Ignacio en Santiago y con la Universidad Pontificia Católica. El Padre Hurtado fue una figura pública. ${ }^{4}$

En este artículo, exploramos la lectura que hicieron de la obra de Dewey los miembros de la Institución Libre de Enseñanza y el Padre Hurtado. Situamos estas lecturas en relación con las ideas políticas, educativas y religiosas de la época, ideas que funcionaron como configuraciones mediadoras con un carácter inestable. ${ }^{5} \mathrm{La}$ intersección con el catolicismo fue relevante en los dos escenarios, aunque las mediaciones fueron sustancialmente diferentes. Ambas lecturas separaron deliberadamente el pensamiento pedagógico de Dewey de su filosofía pragmatista. Sin embargo, mientras el Padre Hurtado hizo una lectura católica para articular el pensamiento pedagógico de Dewey con la trascendencia religiosa, los miembros de la Institución leyeron a Dewey con la intención de liberar a la educación del dogmatismo religioso, pero manteniendo todavía una visión trascendental del ser humano. 


\section{Flujos internacionales, intersecciones y mediaciones en la formación de configuraciones de ideas localmente enraizadas}

\section{Contextos históricos: delineación de los espacios de recepción}

La Institución Libre de Enseñanza fue el centro de uno de los movimientos más importantes de renovación educativa en España en el tránsito al siglo XX. La misma representaba los valores del liberalismo progresista, en sentido político y ético, y estuvo vinculada, de manera más o menos directa, con muchos de los ensayos pedagógicos que intentaron modernizar el sistema educativo. Originalmente, en sus momentos iniciales, el proyecto educativo institucionista se basó en la filosofía de Krause y la pedagogía de Froebel, aunadas en la fórmula de la educación del ser humano como ser humano. La filosofía de Krause había sido introducida en España a mediados del siglo XIX por Julián Sanz de Río. En las generaciones sucesivas el krausismo se convirtió -como escribió el filósofo y pedagogo catalán Joaquín Xirau- más que en un sistema cerrado, en una disciplina moral sustentada en la libertad de investigación y conciencia. En sus filas, decía Xirau, "es posible hallar hegelianos, kantianos, positivistas, ... católicos, librepensadores ... Detesta tan sólo la opaca y anquilosada osamenta de la escolástica decadente." ${ }^{6}$

La introducción del krausismo por Sanz del Río no fue una decisión caprichosa, ni un signo de pobreza intelectual, como durante algún tiempo se mantuvo desde los círculos intelectuales más conservadores, preocupados por la amenaza que éste podía suponer a los valores tradicionales. Más bien, había algunas condiciones que sustentaban esta recepción. Se ha señalado que los elementos humanísticos, místicos e incluso explícitamente religiosos, presentes en el racionalismo armónico de Krause, hacían sus ideas familiares en España. ${ }^{7}$ Solomon Lipp sintetizó bien las condiciones para la recepción cuando escribió: "Los intelectuales españoles, aunque se rebelaban contra lo que consideraban dogmatismo teológico, sentían, sin embargo, la necesidad de llenar un vacío espiritual. El krausismo parecía ser la respuesta: él representaba la razón, la rectitud moral y la libertad, como valores opuestos a lo que estos intelectuales percibían como corrupción, hipocresía y absolutismo." ${ }^{8}$ Los miembros de la Institución vieron en el racionalismo armónico de Krause la puerta a una religiosidad libre y sincera, compatible con un catolicismo abierto, en contacto íntimo con Dios a través del mundo, y un fuerte sentido de humanidad.

La actitud religiosa de los krausistas españoles se vio sacudida por la radicalización conservadora de la jerarquía de la Iglesia Católica durante el papado de Pío IX, expresada en el rechazo a la libertad de conciencia y a la autonomía de la razón en el Syllabus de 1864, y la aprobación del dogma de la infalibilidad del Papa en el Concilio Vaticano I, concluido en 1870. Muchos krausistas, entre ellos Giner de los Ríos, que profesaban un catolicismo antidogmático, compatible con la libertad de conciencia y el pluralismo religioso, vieron imposible conciliar su confianza en la libertad, la razón y el progreso, con la posición de la Iglesia, y se separaron de ella. Abellán subraya la importancia de este momento y su influencia en las generaciones posteriores de intelectuales españoles, cuando escribe: "La crisis krausista es histórica, 
porque supone una crisis de la conciencia española. A partir de ese momento ya no es lícito identificar el pensamiento español con el catolicismo, porque las excepciones van a ser abundantes y, en muchas ocasiones, pertenecen la las cumbres más altas de la cultura o de la filosofía."

Elías Díaz, en su clásico estudio Filosofía Social de Krausismo español, destaca, junto a la base moral indicada, una base política que explicaría también la recepción de la filosofía krausista. Se trata de la exaltación de lo individual, que resultaba muy coherente con el organicismo político del liberalismo progresista español y su rechazo a un intervencionismo estatal excesivo. ${ }^{10}$ Pero la militancia política de los hombres de la ILE no fue uniforme. El escepticismo de los primeros institucionistas acerca de las reformas hechas "desde arriba," fue reemplazado, en las generaciones posteriores, por una actitud más favorable a la intervención política, a veces acompañada de un acercamiento explícito a posiciones socialistas. Éste fue el caso de los dos principales difusores del pensamiento de Dewey, Domingo Barnés y Lorenzo Luzuriaga. Barnés fue dos veces Subsecretario y, durante unos meses, Ministro de Instrucción Pública y Bellas Artes, durante la Segunda República (1931-1936). Desde este puesto, tuvo que desarrollar el principio de escuela laica de la Constitución de 1931. Luzuriaga perteneció a la Liga de Educación Política, fundada por José Ortega y Gasset y Manuel Azaña en 1914, y a la Escuela Nueva de Núñez de Arenas, afín al Partido Socialista Obrero Español. Luzuriaga encarnó el ideario socialista de una escuela única, pública y laica.

La recepción y la lectura que se hizo de Dewey estuvieron condicionadas por el trasfondo histórico de la Institución y su configuración de ideas, fuertemente enraizada en la historia española y europea. La base de humanismo moral de los primeros krausistas españoles, obligará a una lectura suavizada del pragmatismo, por decirlo en términos similares a los empleados por el pensador catalán Eugeni D’Ors para referirse a la lectura que hizo el filósofo francés Émile Boutroux de la obra de William James. ${ }^{12}$ No todos los institucionistas fueron igualmente krausistas, y la influencia de esta filosofía fue desvaneciéndose en el cambio de siglo. No obstante, su influjo seguirá todavía presente en la forma en la que se acercaron a Dewey. Igualmente, el liberalismo de los primeros krausistas dejó paso a un mayor compromiso político, lo que propició, a su vez, una lectura más política y socialmente orientada.

El Padre Alberto Hurtado, miembro de la Compañía de Jesús, y canonizado en Roma, por Benedicto XVI, en 2005, ocupa un lugar importante en el imaginario chileno, como un educador social comprometido con los pobres, la educación de los jóvenes como líderes sociales y la organización de los trabajadores. En 1944 fundó el Hogar de Cristo para niños, jóvenes y adultos sin hogar. En sus años formativos en la Universidad de Lovaina, el Padre Hurtado comprendió la presencia política de las nuevas ideas educativas en Chile y el peso de las propuestas de Dewey. La lectura que hizo de éste, pasó a formar parte de una compleja configuración de ideas desarrollada como respuesta a las visiones del mundo que exponían los socialistas, los comunistas y los representantes del Partido Radical. Éste último surgió en el siglo diecinueve y proclamaba "una postura democrático-liberal inflexible, acentuada más tarde por una nota de ferviente anticlericalismo," 12 fenómeno que tiene mucho que ver con las 
conexiones entre los Radicales y la francmasonería ¿Por qué reorientar la lectura de Dewey hacia la perspectiva católica?

Durante los años veinte y treinta del siglo XX, se extendieron en Chile las nuevas ideas educativas. Éstas fueron adoptadas por la Asociación General de Profesores, que jugó un papel de liderazgo, como actor colectivo, en el cambio de la educación entre 1923 y 1928. En el año 1928 Chile vivió una experiencia única, introduciendo los conceptos de la "nueva pedagogía" en el conjunto del sistema educativo público. La experiencia duró ocho meses. Los líderes de la Asociación se inspiraron en una selección de educadores como Decroly, Kerschensteiner, Dewey, Kilpatrick, Agustín Nieto Caballero, y profesores marxistas que trabajaban en la Unión Soviética, como Pistrack, Pinkevich y Chasky. ${ }^{13}$ Los cambios representaron una "ruptura" drástica que, aunque breve, tuvo repercusiones en el futuro. Entretanto, la crisis socio-política que acompañó a la depresión económica internacional de 1929 dio vida, aunque tan sólo por un corto tiempo, a la "república socialista de Chile." Las rupturas y discontinuidades resultaron tan dramáticas como breves. No menos importante fue que bajo el gobierno del Partido Radical y la Constitución de 1925, la Iglesia y el Estado se separasen, y que el gobierno aceptase pagar un subsidio a la Iglesia. La nueva situación, aunque aceptada con reticencias por la jerarquía clerical, no alteró el lugar que ocupaba la Iglesia Católica en la vida chilena. ${ }^{14}$ Es conveniente tener en cuenta que la influencia de Dewey ganó preeminencia a través de educadores liberales como Darío Salas (quien tradujo el Credo Pedagógico de Dewey en 1908), Irma Salas y Amanda Labarca. Todos ellos tuvieron fuertes conexiones con el Partido Radical (liberal) y con el Teachers' College de Columbia University, en Nueva York. Labarca estableció vínculos con el protestantismo tradicional, por aquel entonces influenciado por el movimiento del evangelio social (social góspel). Darío Salas y Amanda Labarca fueron miembros también de la Asociación Nacional de Educación, la cual abogaba por un reformismo modernista a la vez que nacionalista. ${ }^{15}$ Este acercamiento representaba otra lectura más de Dewey, que no se dio sólo en Chile, como ejemplifica la reforma educativa de los años 1920s and 1930s del México post-revolucionario. La noción deweyana de las comunidades locales y su interacción pedagógica con la gran comunidad facilitó este tipo de articulaciones.

Aunque la Iglesia Católica chilena estaba tradicionalmente ligada a los conservadores, los líderes progresistas católicos vieron la necesidad de prestar atención a la pobreza, la miseria y la explotación dominante en la sociedad chilena. Las enseñanzas sociales de Papas como León XIII y Pío XI expresaban recelos hacia la economía occidental y el orden social imperante, y trataron de desarrollar la tradición comunitarista católica. A principios del siglo XX, la doctrina social de la Iglesia adquirió presencia en Chile. El pensamiento social cristiano inspiró el trabajo de varios líderes de la Iglesia y su acción social. Resulta aquí particularmente relevante el caso del jesuita Fernando Vives del Solar. Vives desarrolló su apostolado social en favor de los pobres, lo cual no fue siempre del agrado de los católicos chilenos tradicionales, de la Compañía de Jesús y del clero y sufrió largos períodos de exilio. Este jesuita ejerció una gran influencia en la formación espiritual de Alberto Hurtado, así como en su compromiso con el movimiento de renovación social del cristianismo que alcanzó un punto culminante en la década de los treinta. ${ }^{16}$ 
Entre 1925 y 1936 Alberto Hurtado, ausente de Chile durante su formación intelectual y religiosa, mantuvo correspondencia con el Padre Vives, Manuel Larrain Errázuriz (futuro obispo de Talca) y otros. Estudió Pedagogía en Lovaina por insinuación del rector del Colegio Máximo de Lovaina, Jean-Baptiste Janssens, S.J., conocido por su compromiso con el apostolado social. ${ }^{17}$ En el contexto del desarrollo de estas ideas sociales, no sorprende que, en la época en la que Hurtado regresaba a Chile, un grupo de disidentes del antiguo partido conservador, cercano a la Iglesia Católica, fundase la Falange Nacional (origen, más tarde, del Partido Demócrata Cristiano), convertido en partido independiente en 1938. Los líderes se inspiraron en las encíclicas sociales del papado y, en particular, en la filosofía social y política de Jacques Maritain. ${ }^{18}$ Les animaba la necesidad de reconciliar el catolicismo con una modernidad liberal. Mientras tanto, la noción de "frente popular" conquistaba Chile, y la alianza de los partidos radical, demócrata, socialista y comunista, junto con la Confederación de Trabajadores, ganaba las elecciones de 1938. Con este telón de fondo, Alberto Hurtado su tesis titulada Le systeme pédagogique de Dewey devant les exigences de la doctrine catholique, defendida en la Universidad Católica de Lovaina, en 1935.

Lovaina era un centro de irradiación pedagógica. La École de de pédagogie et de psychologie appliquée à l'éducation y el Laboratoire de Pédagogie Expérimentale, dieron preeminencia a la pedagogía experimental, interesada por la vertiente "neutral" de la educación y, en particular, los procesos, métodos y medios de enseñanza - los fines de la educación eran competencia de la teología — lo cual facilitó su articulación con las doctrinas católicas. El director de Tesis de Hurtado fue Raymond Buyse, discípulo de O. Decroly con quien trabajó y viajó a Estados Unidos y quien, en 1928, fundó el Laboratoire. ${ }^{19}$ Sin embargo, aunque Hurtado estuvo tan implicado en la metodología científica como su director, su interés se orientaba más hacia la educación como medio de transformación individual y social. ${ }^{20}$

En 1936, inició su acercamiento multi-direccional para cumplir su vocación espiritual apostólica, como sacerdote comprometido con un evangelio social. ${ }^{22} \mathrm{El}$ examen crítico de las ideas de Dewey desde una perspectiva católica, abrió una vía a los católicos para explorar las nuevas ideas en educación. Para Hurtado, fue también una manera de indagar críticamente la visión de lo humano y el carácter redencionista de la educación social cristiana en el contexto de la modernidad. Comprendió la necesidad de reconciliar la tradición católica con las ideas educativas y científicas emergentes, y no rehuyó la relación con los postulados del método científico (articulado por Dewey como un ejercicio sistemático de la inteligencia empírica), la noción de comunidad democrática y la de moralidad social. Nos centraremos en la lectura que Hurtado hizo de Dewey en su Tesis doctoral, con alguna referencia a otros escritos.

\section{Las Lecturas}

Dewey fue leído en España por quienes pretendían reformar la educación, a menudo en oposición abierta a las posturas más cercanas a la Iglesia Católica. Domingo Barnés lo introdujo en España entre los instrumentos conceptuales de la paidología. 
Barnés, perteneció a la generación de jóvenes de la Institución Libre de Enseñanza. Fue uno de los impulsores del estudio de la psicología y pedagogía infantil (paidología), bajo la influencia de la psicología funcional de Claparède. Éste había escrito la introducción a la recopilación de trabajos de Dewey L'École et l'Enfant, traducidos por Pidoux. Esta introducción ha sido considerada el primer estudio en francés de la pedagogía de John Dewey, ${ }^{22}$ y fue parcialmente publicada en español por el Boletín de la Institución Libre de Enseñanza en $1922 .{ }^{23}$ Claparède hacía una lectura psicopedagógica de Dewey, centrada en tres características, genética, funcional y social, defendiendo explícitamente la separación de la pedagogía y la psicología de deweyana de su filosofía pragmatista. ${ }^{24} \mathrm{La}$ trascripción al español del texto en el que Claparède aboga por esta separación, dice:

También desde el punto de vista pragmático, Dewey ha tomado en consideración el importante dominio de la educación, y en ninguna parte como aquí parece tener más bella fecundidad; pues si aplicado a la moral y a la lógica levanta ciertas dificultades, con respecto a la educación, por el contrario, parece que agrupará a todos los espíritus imparciales.

Mas, dejemos el pragmatismo. La psicopedagogía de Dewey, aunque sea la expresión fiel de aquél, no está para nada ligada a la suerte de esta doctrina. El mismo Dewey no pronuncia ni una sola vez esta palabra en toda su obra educativa, y ésta pudo ser muy bien concebida por un psicólogo o un biólogo que no se hubieran preocupado jamás por las cuestiones de filosofía teórica. ${ }^{25}$

Barnés, uno de los principales difusores del pragmatismo y de Dewey en España, mantenía una estrecha relación con Claparède y con el Instituto J.J. Rousseau, que éste había fundado en Ginebra. En 1915 tradujo y publicó en español la conferencia de Dewey "La escuela y el progreso social," ${ }^{26}$ primer capítulo del libro La escuela y la sociedad, que él tradujo y prologó basándose en la introducción de Claparède a L'école et l'enfant. En 1926 volvió a traducir y a prologar otra recopilación de trabajos de Dewey, publicada con el título La escuela y el niño. ${ }^{27}$ Barnés vuelve a referirse aquí a la interpretación de Claparède, asumiendo la disociación que proponía éste entre la psicopedagogía deweyana y la filosofía pragmatista:

Tiene razón Claparède cuando afirma que, aunque la psicopedagogía de Dewey sea la expresión fiel del pragmatismo, su suerte no está de ningún modo ligada con la suerte de esta doctrina. Y es curioso y sintomático que Dewey no la nombre siquiera en toda su obra filosófica. ${ }^{28}$

Lorenzo Luzuriaga fue el segundo gran difusor de las ideas de Dewey en el entorno de la Institución Libre de Enseñanza. En un artículo publicado en el periódico El Sol, en 1918, en el que anunciaba la inminente aparición de su traducción de School of Tomorrow, insistía en el aspecto funcional de la pedagogía de Dewey. Y, más tarde, en la introducción a la edición española de The child and the curriculum (publicada con el título El niño y el programa escolar, en 1925), afirmará que, con excepción de 
la introducción Claparède "no conocemos trabajos de conjunto sobre la pedagogía de Dewey." ${ }^{29}$ Luzuriaga vuelve a recoger aquí las tres características de Claparède, a las que añade las de actividad, vitalidad y reconocimiento de la personalidad de la infancia. Apoya asimismo esta lectura en Kerschensteiner, con cuyas ideas se familiarizó en Alemania en 1913: "John Dewey, puede ser, en efecto, considerado como uno de los creadores de la moderna 'escuela activa'. Nadie como él ha defendido el principio de actividad en la escuela, coincidiendo en este punto con la concepción de Kerschensteiner de la 'escuela del trabajo'." 30

Estas mediciones se articulan con otro tipo de lecturas e intereses. Barnés fue más allá de Claparède en su interpretación social de Dewey, cuya pedagogía, decía, es social "no sólo por ser funcional, como indica Claparède, sino por sus características todas." ${ }^{11}$ Luzuriaga, que puso mucho interés en traducir y hacer circular los trabajos de Dewey, fue incluso más lejos en el empeño por mostrar esta vertiente social y política de sus ideas.

Al igual que Barnés, el Padre Hurtado usó el mismo párrafo de Claparède, junto con otras fuentes, para apoyar su tesis de que el sistema pedagógico de Dewey no estaba fundado en su filosofía pragmática del experimentalismo (como a Hurtado le gustaba llamarla), la cual era incompatible con la doctrina de la Iglesia Católica. Claparède había señalado también en su introducción a L'école et l'enfant que, llevado al dominio de la psicología, el pragmatismo se convertía en el método experimental. ${ }^{32}$ Aún más, Hurtado afirmaba que Dewey desarrolló sus ideas pedagógicas antes de convertirse en pragmatista. ${ }^{33}$ My Pedagogic Creed ocupa un lugar importante en el análisis de Hurtado, aunque no dejó de referirse a las obras de Dewey hasta 1935.

El Padre Hurtado entendía el evangelio como un evangelio social y, al igual que los representantes americanos del protestantismo tradicional que abrazaron la tradición del evangelio social (social gospel), él pensaba en una educación religiosa socializada y en una individualidad socializada. En esto coincidía con los objetivos de la educación moderna. Sin embargo, mientras el pragmatismo no parecía ser un problema para los educadores religiosos protestantes de la época, como George A. Coe, quien integró por completo las ideas de Dewey con el evangelio social. en su búsqueda de una democracia de Dios, para Hurtado esta filosofía sí representaba una dificultad. Él dio al sacerdote un rol mediador en la generación de conciencia social, y rechazó la idea de que el pragmatismo estuviese en la raíz de la educación progresiva y las teorías de Dewey. A pesar de que en el discurso de Hurtado la libertad de conciencia era un elemento central, la posición filosófica de Dewey centrada en la inevitabilidad de la incertidumbre, entraba en conflicto con la concepción de la Iglesia como depositaria de la verdad. El rechazo de Dewey al dualismo no era menos problemático dada su radical importancia para la teología católica de la época, según se expresaba habitualmente en la comprensión dualista del alma y el cuerpo. Para los seguidores protestantes del social gospel, como Coe, la práctica de experimentar la comunión (fellowship) con la totalidad divina, aseguraría el poder moral para lograr nuevos ideales. Para los seguidores del social gospel, la cuestión no resuelta de la trascendencia quedaba suavizada por el énfasis que ponían en la experiencia religiosa y en los principios psicológicos arraigados en las teorías del pragmatismo americano. ${ }^{34}$ 
La lectura que Hurtado hizo de Dewey fue también política, en la línea del humanismo liberal de Jacques Maritain y de los proyectos reformistas político-sociales católicos que ciertos sectores de la Iglesia empezaban a articular en Chile. ${ }^{36}$ Dewey proporcionó a Hurtado una teoría pedagógica que buscaba la transformación social y una crítica al carácter individualista de la educación. Hurtado estaba convencido de que la problemática social en Chile era educativa. ${ }^{36}$ En 1942 escribió que para elevar la situación del pueblo chileno, en particular la de los desposeídos, hacia una visión espiritual de la vida era preciso empezar educándolos. Para Hurtado, la educación tenía una dimensión de redención, que hay que entender en relación con su profunda vocación en favor de los que sufrían, de los pobres y de los jóvenes, tal como revela su trabajo como fundador y trabajador del Hogar de Cristo. No separó la educación de la moralidad social, o, en otras palabras, de las obligaciones hacia los seres humanos, basadas en el mensaje cristiano y en las enseñanzas de la Iglesia. ${ }^{37}$ Está de demás decir que este tipo de discurso hizo a Hurtado vulnerable a las críticas desde dentro de la Iglesia, llegando incluso a ser visto como correa de transmisión de ideas peligrosas. Le creó también tensiones dentro de la Compañía de Jésus. ${ }^{38}$

Hay elementos importantes de las teorías de Dewey que Hurtado vio congruentes con las enseñanzas Católicas, como, por ejemplo, el proceso educativo orientado hacia la vida (afirmó que la educación es vida), el pensamiento reflexivo, el desarrollo de un "espíritu inclinado a la observación," un prudente escepticismo frente a las conclusiones precipitadas y hacia el "dogmatismo exagerado," ${ }^{39}$ la libertad en el aula y el énfasis en la motivación intrínseca. Hurtado consideraba que el entendimiento realista que tenía Dewey de la inteligencia como algo activo y con poder para transformar la realidad, así como el énfasis que éste ponía en la responsabilidad social, eran conceptos muy valiosos. ${ }^{40}$ También reconoció la importancia de la postura deweyiana de la "unión de la vida intelectual y moral" y "el carácter moral de cualquier toda social," que no es el resultado de una voluntad extrínseca al sujeto. No obstante, a su juicio, los fundamentos teóricos que sustentaban estos dos principios eran sólo parcialmente verdaderos (demi vrai) al carecer de la dimensión supranatural. ${ }^{41}$ Desde el punto de vista de Hurtado, la vida moral no debería basarse en una ley totalmente extrínseca al sujeto, una ley que se debe obedecer porque así se manda, incluso si dicha ley no contiene ninguna idea de justicia o su razón de ser no es evidente. ${ }^{42}$ En esta cuestión, su posición era cercana a la de Dewey. Pero Hurtado llevó el argumento aún más lejos, en la línea de su fe católica. En su Tesis escribió que la "actitud moral es intelectual, consciente, y proviene de la luz personal; el deber del profesor es situar al niño en un tipo de ambiente que facilite que la luz brille cada vez más." ${ }^{43}$ Este aspecto es interesante - y no demasiado alejado de Dewey - ya que parece representar un desafío a los modelos dominantes del cristianismo institucionalizado de la época (en especial el católico), en los que la adhesión a las reglas basada en ritos, prohibiciones, observancias y autorizaciones, era acentuada como medio para alcanzar la perfección espiritual. Pero, según decía Hurtado, Dewey fracasó al no reconocer que la luz personal interior, la luz de la razón y de la ley moral, que guía la conciencia, es la manifestación en los seres humanos del orden de cosas creado por Dios. ${ }^{44}$

Hurtado, ponía en paralelo la comprensión del papel del profesor como guía que 
ayuda al niño a adquirir más experiencias y a "descubrir la verdad," con la concepción del profesor como representante de la autoridad de la Iglesia, el Estado, y la familia, agencias todas ellas a las que Dios encarga la misión de educar al niño. En el pensamiento de Hurtado, la práctica del aprendizaje tenía que centrarse en el niño, y las materias debían organizarse siguiendo la psicología del niño. Pero para él había una mediación espiritual en el proceso de aprendizaje. Hurtado trató de articular esta mediación dentro de los parámetros de la Iglesia Católica.

En los dos casos que examinamos aquí, los miembros españoles de la Institución Libre de Enseñanza y el chileno Padre Hurtado, hay una lectura mediada de Dewey, que lleva a una reintegración de las ideas en configuraciones en proceso de formación. El tratamiento de los conceptos de crecimiento, experiencia, y, en particular, de democracia, ejemplifican la separación de la matriz filosófica de Dewey y la "impureza" de su recepción. En el contexto de la convulsa situación española de las primeras décadas del siglo veinte, Barnés y Luzuriaga se asomaron a los escritos de Dewey buscando ideas que apoyasen un proyecto pedagógico, social y político. Pero el trasfondo intelectual histórico les llevó a una lectura que desvinculaba la teoría pedagógica de la filosofía pragmatista. Esta desvinculación facilitaba una lectura trascendentalista de Dewey, en la que el crecimiento se convierte en autorrealización, despliegue. A Barnés esta conversión le permite unir la pedagogía de Dewey con la filosofía krausista, para la cual "realizar la propia esencia era también la finalidad ética del individuo," ${ }^{45}$ y con el naturalismo rousseauniano. Con referencia a éste, escribe: "Lo primero para que lo debe prepararse al niño, dice Rousseau, es para desempeñar su misión de hombre. Y Dewey suscribiría plenamente estas palabras, haciendo únicamente la salvedad de que el hombre es un ser social y sólo en la sociedad, en acción y reacción con ella, puede cumplir su misión y su destino." 46

Estas lecturas suponen desvirtuar la noción deweyana de la educación, en la que no existe ninguna finalidad externa que guíe el proceso, ninguna esencia que realizar, ningún destino que cumplir. Como ha señalado Oelkers, Dewey retiene el término crecimiento, pero le da un significado radicalmente distinto al que tenía en las teorías pedagógicas anteriores, que lo aleja tanto del concepto orgánico de crecimiento como de las concepciones finalistas del desarrollo. ${ }^{47}$ Dewey deja clara esta distinción en el capítulo 5 de su obra Democracia y Educación, traducida por Luzuriaga y publicada en varios volúmenes por la editorial La Lectura entre 1926 y 1927.

La lectura de Dewey en España, y en particular en el espacio de la Institución Libre de Enseñanza, fue una lectura política. Esto es evidente en el caso de Luzuriaga y de su actividad como director de la Revista de Pedagogía, especialmente en los tiempos de la segunda república (1931-1936). Los dos últimos trabajos de Dewey que se publicaron en la revista, antes de su cierre en 1936, fueron "La educación y los problemas sociales," conferencia pronunciada por Dewey en la Asamblea de Inspectores y Directores de Enseñanza de Minneapolis el 1 de marzo de $1933^{48}$ y "El futuro del liberalismo," conferencia dada en la vigésimo cuarta asamblea de la American Philosophical Association, en Nueva York, en diciembre de $1934 .{ }^{49}$

Sin embargo da la sensación de que Luzuriaga tuvo dificultades para integrar esta lectura más política con la lectura pedagógica de Dewey, debido quizás a su rechazo 
a acometer una inmersión en el pragmatismo que le permitiese trazar la necesaria articulación. Acabada ya la Guerra Civil Española, Luzuriaga envió a Dewey, desde el exilio, una copia dedicada de su libro Historia de la Educación pública. ${ }^{50}$ En él considera a Dewey un eminente defensor de la educación democrática en el mundo, y con respecto a España señala que "las primeras manifestaciones a favor de la democratización de la educación y de la escuela unificada fueron las del autor de esta obra, al dar cuenta desde 1914 del movimiento de la Einheitsschule alemana y al difundir las ideas de la educación democrática en diversas publicaciones periódicas." ${ }^{51}$ La democracia se refiere aquí a una dimensión organizativa externa del sistema escolar, no a la dimensión interna, al trabajo del aula como un entorno en el que se plasma la concepción de la democracia como comunidad de investigadores, según propuso Dewey.

En su Tesis, el Padre Alberto Hurtado prestó atención a una realización de la democracia en el aula que permitiese conducir a la transformación personal y social. Su punto de partida era que la educación nueva y las ideas de Dewey sobre los métodos pedagógicos se basaban en descubrimientos acerca de la verdadera naturaleza del niño. ${ }^{52}$ Entendía que el ideal democrático representaba una posición verdaderamente cristiana, desde el momento en que implicaba cambios en las relaciones sociales que favorecían la igualdad de oportunidades y la igualdad económica, así como la tendencia a superar "lo que separa y destruye." ${ }^{53}$ El concepto de cooperación, dominante en la educación progresiva y en el pensamiento de Dewey, y la consideración de la democracia como ideal ético, vienen enseguida a la mente. Sin embargo, Hurtado argumentaba que las ideas democráticas de Dewey estaban estrechamente relacionadas con su filosofía experimentalista, la cual se traducía en la noción de la educación como continua reconstrucción y reorganización de la experiencia que aumentaba su significado social. Esta concepción le resultaba problemática, pues suponía un concepto de crecimiento que excluía la creación y la autoridad trascendental. No dejaba espacio para un tipo de moralidad distinta a la idea del individuo autónomo, y fijaba como única medida de las consecuencias de nuestros actos el criterio pragmatista. Hurtado no podía aceptar esta idea de la democracia porque respondía a una concepción puramente humana de la sociedad, del trabajo humano, y se insertaba en el contrato social sin ninguna referencia al carácter de ley natural que, a su juicio, era intrínseco a la vida social. Aún más, dijo que esta concepción ignoraba el verdadero fin de la vida humana, que es servir a Dios. ${ }^{54}$ Una concepción metafísica de la democracia basada en el experimentalismo no podía ser aceptada por un católico; la integración de los principios pedagógicos prácticos y sociales requería una concepción filosófica distinta. ${ }^{55}$ El agnosticismo y el relativismo fueron problemas evidentes que Hurtado tuvo que enfrentar al tratar las nociones deweyanas de moralidad y moralidad social, esta última situada en el centro de una sociedad democrática. La incorporación de la verdad trascendental chocaba con la posición filosófica de Dewey, según la cual la verdad no puede entenderse en términos absolutos o últimos, sino más bien en el sentido más provisional de una "asertividad garantizada." ${ }^{56} \mathrm{El}$ problema, difícil y no resuelto, surge porque Hurtado vio la oportunidad de utilizar la metodología de Dewey para ayudar a los niños a descubrir la ley moral, pero para él era preciso dejar sitio al valor absoluto de la verdad revelada. 
La búsqueda de una práctica progresiva de la libertad, el desarrollo del pensamiento crítico y la exploración de nuevas experiencias contribuían, según Hurtado, a educar la conciencia y a desarrollar el carácter, siendo aceptables para un católico. Hurtado escribió que, aunque en ocasiones se olvide, los aspectos individual, intelectual y racional de nuestra vida moral tienen un gran valor. Fue aún más lejos al decir que la mediocre educación moral de muchos católicos se basaba en un código prescriptivo, externo a ellos, más que en el desarrollo de la luz interior que se proyecta sobre las acciones de las personas. La diferencia con Dewey es que esa luz de la razón, que para Hurtado es la ley moral, es la manifestación a los seres humanos del orden de cosas creado por Dios. ${ }^{57}$ La crítica de Hurtado a los límites de la espiritualidad institucionalizada se pone una y otra vez de manifiesto.

En España, Dewey encontró resonancia porque sus escritos confluían con las aspiraciones de la época, en particular, sus ideas acerca de la reforma de la educación, pero también, hasta cierto punto, la propia base pragmatista de su pedagogía. En el caso de la Institución Libre de Enseñanza no puede hablarse estrictamente de un anti-pragmatismo. Más bien existía una actitud ambivalente. Los representantes de la Institución, como intérpretes intelectuales de su época, expresaron bien esa postura vital, a la que se refería James en 1906 y 1907, de quienes "quieren un sistema que combine ambas cosas, la lealtad científica a los hechos y la disposición a hacerse cargo de ellos, en suma, el espíritu de adaptación y acomodación, pero también la vieja confianza en los valores humanos y en la espontaneidad correspondiente, sea de tipo religioso o romántico." ${ }^{58} \mathrm{El}$ pragmatismo fue la respuesta de James a esta tensión entre lo que él llamó espíritus rudos y selectos; el krausopositivismo, un intento de combinar racionalismo y experiencia, fue la respuesta de los institucionistas españoles.

Dentro de esta tensión, el pragmatismo de Dewey se inclina hacia el mundo empírico, y por ello, como señaló Rorty, al contrario de lo que sucede en James, para él la religión no constituye una fuente válida de conocimiento. ${ }^{59}$ Los institucionistas españoles defendieron una educación neutral, laica, lo que les enfrentó con la Iglesia Católica. Sin embargo, había una diferencia sustancial con Dewey, porque su proyecto pedagógico requería una noción de humanidad que fuese más allá de las condiciones dadas. De aquí que acometiesen una lectura trascendentalista de Dewey.

La intersección de la religión era inevitable en la lectura que Hurtado hizo de Dewey. Esta intersección tenía una dimensión política e histórica, dada la historia de Chile y su gente, pero la lectura se hizo desde una perspectiva confesional con intencionalidad histórica. Para Hurtado, la separación de la religión y de la experiencia religiosa era un paso más en la dirección hacia la negación de la posibilidad de un conocimiento intelectual y verdadero de Dios (aunque Hurtado reconoció que éste era un conocimiento imperfecto), y la negación de las doctrinas relacionadas con la espiritualidad y la inmortalidad del alma. La Iglesia Católica dejaba claro que Dios sólo puede conocerse a través de la razón natural.

La cuestión de la trascendencia y el lugar de lo trascendente en la vida social estaban en el centro de la divergencia de posiciones. Dewey era un naturalista, mientras que la visión de Hurtado era teo-céntrica, con la condición de que lo sobrenatural complementa la naturaleza. A mediados de los años 1930 y 1940, Hurtado escribió 
artículos sobre Dewey y también sobre la Educación Nueva con algunas variaciones en su enfoque. En 1947, preocupado por la enseñanza de la religión, propuso un plan que contenía principios pedagógicos innovadores para la Iglesia Católica chilena. El primer principio era el énfasis puesto en los componentes dinámicos y funcionales de la verdad religiosa, más que en los estáticos y estructurales. Era importante mostrar la renovación interna de los dogmas. El segundo principio era ser consciente de los peligros de hacer de la enseñanza religiosa una enseñanza de moralidad basada en mandamientos separados de la teología. La práctica de la vida se relacionaría con los principios teológicos. El tercer principio era que el estudiante se situase en un vis a vis con la vida sobrenatural, lo cual no sólo se realizaba en el cielo ni se confinaba al alma. En lugar de eso, la vida sobrenatural debía ser vivida en la tierra, incluyendo al ser humano por completo e irradiándose en la vida social. El cuarto principio era la toma en consideración de la psicología del estudiante. ${ }^{60}$ En el mismo artículo, Hurtado escribió que "una clara comprensión de la elevación de la naturaleza a través de la gracia ayudaría al estudiante a ver cómo los valores humanos, la libertad de la persona, la unidad social de la raza humana, sólo pueden realizarse en plenitud en la vida sobrenatural, en la unidad de los seres humanos [hombres en el original] con Dios." 61

El filósofo Daniel Tröhler ha mantenido la hipótesis de que en la obra de Dewey $A$ Common Faith, la humanidad forma una comunidad que está constituida, no por la creencia de base trascendental de que todos somos hijos de Dios, sino por una "totalidad misteriosa del ser," el "Universo." Tröhler continúa diciendo que "esto está conforme con la afirmación antropológica de que el ser humano posee, o ha desarrollado, una capacidad especial para orientarse hacia la participación y la simpatía, la justicia, la igualdad y la libertad." ${ }^{62} \mathrm{Al}$ superar la dicotomía entre la visión sobrenatural y el "mecanicismo," la "comunidad comprensiva" de Dewey supo dar con los requisitos para una democracia ideal. ${ }^{63}$ Tröhler asocia este punto de vista con los supuestos religiosos latentes en el fondo de la filosofía social de Dewey y que, siguiendo el argumento de Tröhler, éste desarrolló explícitamente en A Common Faith. Una pregunta decisiva a este respecto es si Dewey tenía todo lo que necesitaba en su teoría de la experiencia para desarrollar su noción de comunidad comprensiva. Si la respuesta es afirmativa, no cabría discutir acerca de supuestos religiosos. ${ }^{64}$ Es importante señalar aquí que Hurtado vio en la filosofía de Dewey una divinización del ser humano; el humano tomó el lugar de Dios; en lugar de en la religión, situó abiertamente la fe en el poder creativo de la inteligencia. ${ }^{65}$ A primera vista, parece haber un punto de convergencia entre la noción de "comunidad comprensiva," tal y como la ha analizado Tröhler, y el intento de Hurtado de articular "la unidad social de la raza humana" incorporando la trascendencia desde una perspectiva católica. Ambas perspectivas implican una persona educada de manera integral. Sin embargo, la diferencia reside en el rechazo deweyano de las doctrinas metafísicas y/o trascendentales que pudieran obstaculizar la democracia, debido en parte a concepciones de la superioridad basada en la verdad, particularmente en la verdad religiosa. De otro modo, habría que defender el carácter trascendental de la noción de comunidad comprensiva como "una totalidad mística," un concepto utilizado por Tröhler. 


\section{Conclusión}

A menudo las ideas de Dewey fueron separadas de sus bases filosóficas e integradas en discursos políticos, filosóficos y teológicos, convirtiéndose así en parte de configuraciones de ideas localmente arraigadas. Las lecturas pedagógicas y políticas dieron lugar a interpretaciones "mutiladas" pero vivas de la obra de Dewey, que generaron nuevas variantes de la educación progresiva.

En Experiencia y Educación (1938) Dewey, el autor, reaccionó contra las lecturas parciales de su obra, afirmando que sus ideas pedagógicas no eran simplemente un conjunto de prácticas para ser aplicadas. El siguiente párrafo es ilustrativo:

\footnotetext{
"Creo que basta con un ligero conocimiento de la historia de la educación para probar que todos los reformadores e innovadores de ésta han sentido la necesidad de una filosofía de la educación. Quienes seguían adheridos a los sistemas establecidos, necesitaban tan sólo unas pocas palabras que sonaran bien para justificar las prácticas existentes. El trabajo real se realizaba con hábitos tan fijos que se habían hecho institucionales. La lección para la educación progresiva es que ésta requiere de manera urgente, incluso más apremiante de lo que lo fue para los innovadores anteriores, una filosofía de la educación basada en una filosofía de la experiencia." ${ }^{66}$
}

La cuestión aquí es que cualquier recepción de ideas filosóficas, y sus correspondientes pedagógicas, debido a la traslación y a las inevitables disonancias de las experiencias que las sustentan, tiene que generar resultados "impuros," en función de de los elementos configuradores contextuales que son ajenos a sus formulaciones originales. Sin duda podemos decir que las lecturas de los trabajos de Dewey fueron "impuras" en los espacios de recepción de que nos hemos ocupado en este trabajo.

El tema religioso está presente en los dos casos examinados. La lectura que Hurtado hizo de Dewey, un autor rechazado por la jerarquía de la Iglesia, proporcionó a los católicos una forma de incorporar las ideas progresistas sobre educación, en un país donde dichas ideas formaban parte del imaginario político secular. Los miembros de la Institución Libre de Enseñanza leyeron a Dewey teniendo como fondo el debate sobre la religión y el poder de la Iglesia Católica en la educación. En el caso de Hurtado, la noción de trascendencia se basaba en la espiritualidad católica y en la doctrina de la Iglesia; en el caso de la Institución, lo hacía en la historia intelectual española y de la propia Institución, incluyendo su oposición a las tendencias autoritarias conservadoras de la Iglesia Católica.

Hurtado y los miembros de la Institución Libre de Enseñanza, hicieron un esfuerzo por separar la pedagogía de Dewey de sus fundamentos filosóficos. En el caso de Hurtado, esta disociación se realizaba dentro de la visión dominante en Lovaina de separación de los medios y de los fines, aunque su lectura estuvo impregnada de su 
compromiso con la transformación personal y social, y de la apertura intelectual para articular ideas y fines educativos. En el caso de los autores españoles, esta separación respondía a las exigencias de un concepto de educación que no podía dejar de lado la consideración del ser humano como el "único ser finito capaz de aspirar al infinito." 67

Es significativo que, en una fecha tan tardía como 1948, con ocasión de la publicación en México de la versión española de Experience and Nature, el filósofo español José Gaos, lamentase la falta de interés por traducir a la lengua hispana la obra filosófica de Dewey. Gaos interpretaba esta falta de interés como una deficiencia del mundo hispanohablante. El pragmatismo de Dewey personificaba el desafío de la modernidad, que para Gaos era, en última instancia, la relación entre inmanencia y trascendencia. Dewey, decía Gaos, intentó hacer frente a la brecha entre estos dos mundos naturalizando a la humanidad y a Dios. ${ }^{68}$ Hurtado no pudo aceptar esta solución. Tampoco pudieron hacerlo los miembros de la Institución. Iba demasiado lejos para ellos. La Institución defendía una escuela laica, pero su propuesta pedagógica compensaba la falta de una base confesional explícita con una visión trascendental del ser humano.

Las lecturas que Hurtado y los miembros de la Institución Libre de Enseñanza hicieron de la obra de Dewey, revelan las dificultades para repensar, en términos modernos, un concepto de educación arraigado en siglos de tradición. Dewey entendió bien estas dificultades cuando, en 1909, escribió en The Influence of Darwinism on Philosophy: "Las viejas ideas se abren paso lentamente; ya que son algo más que categorías y formas lógicas abstractas. Son hábitos, predisposiciones, actitudes profundamente arraigadas de aversión y preferencia." ${ }^{69}$ Los dos escenarios muestran no sólo que los hábitos y los sistemas de creencias establecen límites a lo que puede ser integrado en un concepto de educación, sino también la relevancia de las condiciones contingentes en la articulación de las teorías y las prácticas educativas. La educación, quizás más que cualquier otra esfera de actividad, ilustra que la ciencia y la modernidad no pueden suprimir lo que Charles Taylor ha interpretado como "la necesidad de articular un sentido de algo más completo, más profundo." ${ }^{70}$ La necesidad de repensar de nuevo la educación más allá de la contingencia y las tradiciones, conduciría a una ruptura y a una insostenible situación ahistórica.

\section{Notas}

* Nuestro agradecimiento a María Josefina Silva, estudiante en la Universidad Pontificia Católica de Chile, y a María de los Ángeles Ruiz-Valdepeñas, de la Universidad Complutense de Madrid, que trabajaron como investigadoras ayudantes reuniendo fuentes para esta investigación. Nuestro agradecimiento también a Bianca Thoilliez, de la Universidad Complutense, que trabajó en la traducción de borradores previos escritos en inglés y en la preparación del texto final para su publicación.

1. Antón Donoso, "John Dewey in Spain and Spanish America," International Philosophical Quarterly 41, no. 3 (2001): 357. 
2. Un importante punto de referencia es el trabajo de Andrés Guzmán Traversa y Cecilia Ramírez Venegas, "El Pensamiento Educacional del Padre Hurtado en el Contexto de las Ideas Educacionales en Chile durante la Primera Mitad del Siglo XX. "Un Primer Avance," Boletín de Investigación Educacional 16, Facultad de Educación, Santiago, Chile (2001): 15-52.

3. Trasciende la intención de este artículo examinar el desarrollo de la psicología y la teoría de la educación en Lovaina. Puede verse al respecto: George Meuris, "Looking Back: The Beginnings of a Scientific Outlook in Psychology and Education Theory," International Review of Applied Psychology 34 (1985): 7-16; Marc Depaepe, "The Practical Professional Relevance of Educational Research and Pedagogical Knowledge from the Perspective of History: Reflections on the Belgian Case in its International Background," European International Research Journal 1, no. 2 (2002): 360-79.

4. Véase, por ejemplo, Alberto Hurtado, "Psicología Pedagógica: Formación de un Plan de Vida," Revista Católica (Chile) 71, no. 821 (noviembre de 1936): 529-33; Alberto Hurtado, "Psicología Pedagógica: La Formación Intelectual Según Dewey," Revista Católica (Chile) 71, no. 818 (octubre de 1936); Alberto Hurtado, "Psicología Pedagógica: PsicoPedagogía de la Afectividad en la Adolescencia," Revista Católica (Chile) 71, no. 822 (diciembre de 1936): 578-87; Alberto Hurtado, "Psicología Pedagógica: La Escuela Nueva y el Aspecto Social de la Educación," Revista Católica (Chile) 71, no. 816 (septiembre de 1936): 217-26; Alberto Hurtado, "Psicología Pedagógica: La Educación Nueva," Revista Católica (Chile) 71, no. 815 (agosto de 1936): 160-69; Alberto Hurtado, "Psicología Pedagógica: La Educación Nueva," Revista Católica (Chile) 71, no. 814 (agosto de 1936): 111-21; Alberto Hurtado, "Psicología Pedagógica: El Verdadero Concepto del Amor," Revista Católica (Chile) 72, no. 826 (marzo de 1937): 100-06; Alberto Hurtado, "Psicopedagogía de la Afectividad en la Adolescencia," Revista Católica (Chile) 72, no. 825 (febrero de 1937): 87-97; Alberto Hurtado, "La Enseñanza de la Religión en el Segundo Ciclo de Humanidades," Revista Católica (Chile) n. 932 (mayo-junio de 1947). Para las notas de clase y otras obras no publicadas, ver: Una Verdadera Educación: Escritos sobre Educación y Psicología del Padre Alberto Hurtado, S.J., ed. Violeta Arancibia C. (Santiago, Chile: Ediciones Universidad Católica de Chile, 2005). Puede verse también, Escritos de San Alberto Hurtado (Santiago, Chile: Centro de Estudios San Alberto Hurtado de la Pontificia Universidad Católica de Chile, CD-ROM, 2007).

5. Véase Patricio Miranda Rebeco, "Un Conflicto de Interpretaciones: La Figura Intelectual de Alberto Hurtado," Humanitas X, no. 39 (invierno de 2005): 522-35.

6. Joaquin Xirau, "Julián Sanz del Río y el krausismo español," Cuadernos Americanos XVI (1944): 62. Citado en: Elías Díaz, La filosofía social del krausismo (Madrid: Edicusa, 1973), 51-52.

7. Díaz, Filosofía Social, 17-22.

8. Solomon Lipp, Francisco Giner de los Rios: A Spanish Socrates (Waterloo, Ontario: Wilfrid Laurier University Press, 1985), 9.

9. José Luís Abellán, Historia crítica del pensamiento español, vol. 4 (Madrid: EspasaCalpe, 1984), 455.

10. Díaz, Filosofía Social, 22-23.

11. Eugenio D’Ors, Comentario al libro de Émile Boutroux William James (Paris: A. Colin, 1911), Arxius de l'Institut de Ciencies I, n. 1 (1911): 151-52. Reeditado en Eugenio D’Ors, La Filosofia del Hombre que Trabaja y que Juega (Madrid: Libertarias/Prodhufi, 1995), $116-21$.

12. Simon Collier y W. F. Sater, A History of Chile 1808-2002 (Cambridge: Cambridge University Press, 2004), 117. Para una buena comprensión del contexto, véase Elena Sánchez 
Correa y Lucía Díaz del Valle, "El Padre Hurtado: Su Contribución al Fortalecimiento de la Educación Nacional,” Pensamiento Educativo 34 (junio de 2004): 321-54.

13. Iván Núñez Prieto, "El Pensamiento de un Actor Colectivo: Los Profesores Reformistas de 1928," Pensamiento Educativo 34 (junio de 2004): 162-78 y 213.

14. Collier y Sater, History of Chile, 213.

15. Andrés Baeza Ruz, "La Dimensión Educacional de la Crisis del Centenario en Chile: Modernización, Nacionalismo y Reforma” (Tesis para optar al grado de Licenciado en Historia. Facultad de Historia, Geografía y Ciencia Política, Instituto de Historia, Universidad Pontificia Católica de Chile. Santiago, Chile, 3 de mayo de 2006); Emma S. Salas Neumann, El Pensamiento de Dario Salas a Través de Algunos de sus Escritos (Santiago, Chile: Ediciones de la Universidad de Chile, 1987); Julio César Jovet, Doctrina y Praxis de los Educadores Representativos Chilenos (Santiago, Chile: Editorial Andrés Bello, 1970).

16. Véase Jaime Caiceo Escudero, Dios y el Hombre en el Pensamiento Catolico Chileno: 1900-1950 (Pontificia Universidad Católica de Chile, Facultad de Educación, 1992).

17. Caiceo Escudero dice que Hurtado studió pedagogía en obediencia a sus superiores. Sin embargo, Padre Samuel Fernández de la Facultad de Teología, Pontificia Universidad Católica de Chile, compartió conmigo un documento escrito por el Padre Hurtado que dice claramente que el Padre Father Janssens le sugirió que estudiara pedagogía. Vea Curriculum Vitae del Padre Hurtado, [1936], APH s62y053. Está escrito por Hurtado en tercera persona. Jaime Caiceo Escudero, El P. Alberto Hurtado y John Dewey: Presencia Educativa de su Pensamiento en Chile (Pontificia Universidad Católica de Chile, Facultad de Educación, 1986).

18. Collier y Sater, History of Chile, 227; Jaime Caiceo Escudero, Maritain. La Politica y el Social Cristianismo en Chile (Santiago, Chile: Pontificia Universidad Católica de Chile, 1994).

19. Arthur Gille, "Raymond Buyse, Promoteur de la Pédagogie Expérimentale," en L'Oeuvre Pedagogique de Raymond Buyse, Anna Bonboir ed. (Louvain/Brussels: Vander, 1969), 19-358; Marc Depaepe, "The Practical and Professional Relevance of Educational Research and Pedagogical Knowledge from the Perspective of History: Reflections on the Belgian Case in Its International Background," European Educational Research Journal 1, no. 2 (2002): 360-79.

20. Véase Tom DeCoster, Marc Depaepe, Frank Simon, y Angelo Van Gorp, "Dewey in Belgium: A Libation for Modernity?," en Inventing the Modern Self and John Dewey: Modernities and the Traveling of Pragmatism in Education, Thomas S. Popkewitz ed. (New York: Palgrave Macmillan, 2005), 86-109.

21. Véase Samuel Fernández Eyzaguirre, "El Padre Alberto Hurtado, S. J. y la Pontificia Universidad Católica de Chile: Un Santo Universitario,” Humanitas X, no. 39 (invierno de 2005): 458-54.

22. Daniel Tröhler, "Langue as Homeland: The Genevan Reception of Dewey in the Challenge of Modernity," en Inventing the Modern Self and John Dewey: Modernities and the Traveling of Pragmatism in Education, Thomas S. Popkewitz ed. (New York: Palgrave Macmillan, 2005), 68.

23. Edouard Claparède, "La pedagogía de J. Dewey," Boletín de la Institución Libre de Enseñanza XLVI, no. 753 (1922): 353-61.

24. Esta posición había sido ya mantenida por el psicólogo y educador de Zurich, Gustav Störring. En el Tercer Congreso Internacional de Filosofía que tuvo lugar en la Universidad de Heidelberg, en septiembre de 1908, Störring reconoció la importancia del pragmatismo a lo sumo "para la cuestión de la psicogénesis del pensamiento, pero no en un contexto de lógica epistemológica." Citado en Jürgen Oelkers "Remarks on the 
Conceptualization of John Dewey's Democracy and Education" (Lecture for the Annual John Dewey Society Symposium, American Educational Research Association Annual Meeting, Montreal, 11 de abril de 2005), 2.

25. Edouard Claparède, "La Pedagogía de J. Dewey," Boletín de la Institución Libre de Enseñanza XVLI, no. 753 (1922): 354.

26. John Dewey, "La escuela y el progreso social," Boletín de la Institución Libre de Enseñanza XXXIX, no. 662-33 (1915): 129-34 y 161-65.

27. La compilación de Barnés era diferente de la colección publicada por Claparède con el mismo título. El prólogo también fue publicado en el Boletín de la Institución Libre de Enseñanza. Domingo Barnés, "La Pedagogía de J. Dewey," Boletín de la Institución Libre de Enseñanza L, no. 797 (1926): 238-47.

28. Barnés, "La Pedagogía de J. Dewey,” 240.

29. Lorenzo Luzuriaga, "La pedagogía de John Dewey," en John Dewey: El niño y el programa escolar (Madrid: Publicaciones de la Revista de Pedagogía, 1925), 7.

30. Ibid., 14.

31. Barnés, "La Pedagogía de J. Dewey," 243.

32. John Dewey, L'école et l'enfant. Traduit par L.S. Pidoux, Introduction par E. Claparède (Paris: Éditions Delachaux \& Niestlé, 1931), 10.

33. John Shook cuestionó convincentemente esta línea de pensamiento. Demostró que en 1891, cinco años antes de que escribiese, "My Pedagogic Creed," Dewey tenía todas las piezas preparadas para su instrumentalismo. Comunicación de Scott Johnston, 21 de marzo de 2008.

34. George A. Coe, A Social Theory of Religious Education (New York: Charles Scribner's Sons, 1927). La primera edición de la obra es de 1917. Ver Donald Meyer, The Protestant Search for Political Realism: 1919-1941 (Middletown, CT: Wesleyan University Press, 1988, 2a ed.), 141.

35. Véase la citada obra de Jaime Caiceo Escudero. Maritain.

36. Alberto Hurtado, Puntos de Educación (Santiago, Chile: Editorial Splendor, 1942). Este libro está incluido en la citada recopilación Escritos de San Alberto Hurtado.

37. El Padre Hurtado entendió Acción Católica como una iniciativa poseedora de un importante papel en la educación popular. En palabras de Hurtado, sería hacer una caricatura de Acción Católica limitar su trabajo a promocionar comentarios de las lecturas del Evangelio y a mantener prácticas religiosas en grupo. Hurtado, Puntos de Educación.

38. Samuel Fernández analizó los conflictos con la Companía que Hurtado tuvo muy temprano a su regreso a Chile a la luz del espíritu de Lovaina, en contrase con los puntos de vista Espanoles conservadores que dominaban entre los Jesuitas Chilenos. Samuel Fernández, "Los Primeros Conflictos del Padre Hurtado y 'el Espíritu de Lovaina," Teología y Vida LI (2010): 609-26. Vea también Elena Sánchez C. y Lucía Díaz del V., Padre Alberto Hurtado, S.J. La Riqueza de su Pensamiento. (Santiago, Chile: Ediciones de la Universidad Catolica de Chile, 2005), 13.

39. Alberto Hurtado, S.J., Le système pédagogique de Dewey devant les exigences de la doctrine catholique (Belgium: Université de Louvain, 1935), 105.

40. Ibid., 104.

41. Ibid., 195.

42. Ibid.

43. Ibid. 
44. Ibid., 197

45. Barnés, "La Pedagogía de J. Dewey," 240.

46. Ibid., 243.

47. Oelkers, "Remarks on the Conceptualization," 9.

48. John Dewey, "La educación y los problemas sociales actuales," Revista de Pedagogía XII, no.140 (1933): 327-44. $126-31$.

49. John Dewey, "El futuro del liberalismo," Revista de Pedagogía XIV, no. 157 (1935):

50. The Correspondence of John Dewey, 1871-1952. Edición electrónica. Nota a la carta de 28 de agosto de 1942 (referencia 09852). Consultada en el Center for Dewey Studies, Carbondale, IL, el 26 de Julio de 2006.

51. Lorenzo Luzuriaga, Historia de la Educación pública (Buenos Aires: Losada, 1964), 147. Edición original de 1946.

52. Hurtado, Le système pédagogique, 209.

53. Ibid., 185.

54. Ibid., 100.

55. Ibid., 186.

56. Véase Rosa Bruno-Jofré y Martin Schiralli, "Teaching History: A Discussion of Contemporary Challenges," Encounters on Education/Encuentros sobre Educación/Rencontres sur l'Éducation, 3 (otoño de 2002): 117-28.

57. Hurtado, S.J., Le système pédagogique, 196-97.

58. William James, Pragmatism, a new name for some old ways of thinking. Popular lectures on philosophy (New York: Longmans, Green, and Co., 1908), 20.

59. Richard Rorty, Esperanza o conocimiento. Una introducción al pragmatismo (México: F.C.E., 2001), 31.

60. Alberto Hurtado, "La Enseñanza de la Religión en el Segundo Ciclo de Humanidades,” 1016-17.

61. Ibid., 1017.

62. Daniel Tröhler, "The Global Community, Religion, and Education: The Modernity of Dewey's Social Philosophy," Studies in Philosophy of Education 19 (2000): 179.

63. Ibid.

64. Comunicación de Scott Johnston, 21 de marzo de 2008.

65. Hurtado, Le système pédagogique, 103.

66. John Dewey, Experience \& Education (London and New York: Collier MacMillan Publishers, 1963), 29.

67. Domingo Barnés, "Un aspecto de la filosofía de los valores y la pedagogía," en Ensayos de pedagogia y filosofia (Madrid: La Lectura, 1920), 45-46.

68. José Gaos, "Prólogo del traductor," en John Dewey, La experiencia y la naturaleza (México: F.C.E., 1948), XIX-XXXV.

69. John Dewey, "The Influence of Darwinism on Philosophy" en James and Dewey on Belief and Experience, J. M. Capps y D. Capps eds. (Urbana and Chicago: University of Illinois Press, (1909/2005), 188.

70. Charles Taylor, A Secular Age (Cambridge and London: The Belknap Press of Harvard University Press, 2007), 391. 